\title{
Retrospective Review of Patient Self-Reported Improvement and Post-Procedure Findings for mild $@$ (Minimally Invasive Lumbar Decompression)
}

\author{
Richard Lingreen, $\mathrm{MD}^{1}$ and Jay S. Grider, DO, $\mathrm{PhD}^{2}$
}

From: ${ }^{1}$ Frankfort Regional Medical Center, Frankfort, KY; and '2University of Kentucky, Lexington, KY.

Dr. Lingreen is Medical Director of Pain Services, Frankfort Regional Medical Center, Frankfort, KY. Dr. Grider is Chief of Regional

Anesthesia and Pain Medicine; Medical Director of Interventional Pain Associates; and Associate Professor of the Department of Anesthesiology,

College of Medicine, University of Kentucky, Lexington, KY.

Address correspondence: Jay S Grider. DO, PhD Associate Professor Department of Anesthesiology College of Medicine University of Kentucky Lexington, $\mathrm{KY}$

E-mail: jsgrid2@email.uky.edu

Disclaimer: There was no external funding in the preparation of this manuscript. Conflict of interest: None.

Manuscript received: 08/20/2010 Revised manuscript received: 09/28/2010 Accepted for publication: 10/22/2010

Free full manuscript: www.painphysicianjournal.com
Background: Lumbar spinal stenosis and neurogenic claudication functionally impact thousands of patients per year. Those who fail conservative therapies and are not surgical candidates due to co-morbid conditions have few interventional options available. The recently described mild® procedure (Minimally Invasive Lumbar Decompression) is a candidate to fill this void. While 2 studies have reported no major adverse events with this procedure, the typical post-procedure patient course has not been previously described.

Objective: To examine the minor adverse events and periprocedural course associated with mild. Additionally, to evaluate the efficacy of the procedure with regard to pain relief and functional status.

Design: Retrospective evaluation.

Methods: Forty-two consecutive patients meeting magnetic resonance imaging (MRI) criteria for mild underwent the procedure performed by 2 interventional pain management physicians working at the same center. The pre and post procedure visual analog scale (VAS) as well as markers of global function were recorded. Major and minor adverse events were tracked and patient outcomes reported

Results: There were no major adverse events reported. Of the minor adverse events, soreness lasting 3.8 days was most frequently reported. No patients required overnight observation and only 5 required postoperative opioid analgesics. Patients self-reported improvement in function as assessed by ability to stand and ambulate for greater than 15 minutes, whereas prior to the procedure $98 \%$ reported significant limitations in these markers of global functioning. Visual analog pain scores were significantly decreased by $40 \%$ from baseline. Eighty-six percent of the patients reported that they would recommend the mild procedure to others.

Conclusions: The mild procedure appears to be a safe and likely effective option for treatment of neruogenic claudication in patients who have failed conservative therapy and have ligamentum flavum hypertrophy as the primary distinguishing component of the stenosis.

Key words: Minimally invasive lumbar decompression, lumbar spinal stenosis, neurogenic claudication, fluoroscopy, ligamentum flavum

Pain Physician 2010; 13:555-560
$\Delta$ cquired lumbar spinal stenosis (LSS) functionally impacts significant numbers of Americans per year (1). A radiology based survey attempting to establish anatomic criteria for relative and absolute LSS estimated the prevalence of this disorder in patients ranging from 60 to 69 years of age to be as high as $47 \%$ for relative stenosis and $19.7 \%$ for absolute stenosis (2). These data suggest that millions of patients have the potential to be affected by progressive, acquired LSS during the course of their lifetime. It has been aggressively estimated that approximately two-thirds of these patients are 
candidates for open laminectomy or laminotomy with corresponding improvement in function noted, however in this age group many potential candidates from a surgical standpoint are at increased risk in the perioperative period (3). These remaining non-surgical patients are treated with a variety of conservative measures to include lumbar epidural steroid injections, oral opioids, adjunctive medication, implantable therapies such as spinal cord stimulation, and intrathecal drug delivery (4-8). While these therapies have demonstrated varying degrees of efficacy, there are limitations to each modality. For instance, injections might provide only temporary results or might be contraindicated in those who cannot withdraw from chronic anticoagulation therapy. Spinal cord stimulation and intrathecal drug delivery are surgical interventions with varying lengths of recovery required and efficacy for spinal stenosis has not been clearly demonstrated $(7,8)$. Oral opioids and adjuncts might be effective for some patients, however in others they might be poorly tolerated or provide diminishing relief (9-11). Recently a new minimally invasive method to provide relief of neurogenic claudication symptoms in patients with LSS secondary to ligamentum flavum hypertrophy has been described (6).

Deer and Kapural (6) summarized the procedural technique and provide safety data in 90 patients undergoing mild (Vertos Medical, Aliso Viejo, CA). In this report, no major adverse events such as dural tears, cerebrospinal fluid leaks, epidural abscess/hematoma or infection were encountered. The major adverse event focus of that study provided the first data suggesting that this procedure is safe in comparison to open laminectomy/laminotomy. In addition to the safety profile, the limited invasiveness of the procedure is also highlighted by the fact that it is often performed on an outpatient basis with monitored anesthesia care for sedation (6).

With regard to efficacy, the MiDAS 1 trial (mild Decompression Alternative to Open Surgery) reported prospective data from 75 patients undergoing this procedure (12). The effectiveness of the procedure was underscored by increases in several assessments of functional capacity as well as decreases in Visual Analog Pain scores. Like Deer and Kapural, no major adverse events were reported in the MiDAS 1 study. Taken together these preliminary reports suggest the gross safety and likely efficacy of mild $(6,12)$. To date there has been no data with regard to the minor adverse events or attempts to characterize the typical post-procedure course for patients undergoing mild $(6,12)$. Therefore the aim of the current study is to fill important gaps in this emerging body of literature concerning Minimally Invasive Lumbar Decompression or mild. Additionally, we analyzed patient self-reported major adverse events, non-standardized functional assessment and VAS in single-center multi-physician retrospective review.

\section{Methods}

Forty-two consecutive patients $(n=42)$ ages 5286 , with spinal stenosis and ligamentum flavum hypertrophy as the primary feature on magnetic resonance imaging were identified. The patients meeting these criteria underwent the mild procedure performed by 2 anesthesiology-based interventional pain management physicians trained in the technique. All patients had undergone previous conservative treatment to include lumbar epidural steroid injections, opioid and non-opioid medication and physical therapy. Additionally, most had been deemed as non-surgical candidates at that time in consultation with or referral from a spine surgeon. Their clinical responses were evaluated via a retrospective, IRB approved review of outcomes 30 days following the procedure. Patient self reported VAS, pre and post procedure functional assessments of activities of daily living (ADL), major and minor complication reports and need for follow-up procedures were evaluated. Patients were contacted on post-procedure days 3,7 and 14. Additionally, post procedure opioid requirement and responses to patient satisfaction questions are reported.

Prior to data analysis, an improvement of greater than 3 (>3) on the VAS was selected as clinically significant. This is in keeping with other studies on pain efficacy in which a decrease in VAS was determined to be relevant $(12,13)$. Additionally, we determined that the majority of the patients self-reported that ambulation and standing for greater than 15 minutes was significantly impeded by neurogenic claudication symptoms secondary to LSS.

Other non-parametric data such as the number of days affected by minor adverse events, post-procedure opioid requirements, and patient satisfaction scores were also evaluated. Data were also obtained with regard to the number of levels required based on MRI criteria and their anatomic spinal levels.

Statistical evaluation of non-parametric data (VAS) was performed using the Mann-Whitney $U$ test (14) with significance set prior to data analysis at $P<0.05$. Other data are not evaluated statistically but are reported descriptively. 


\section{Results}

A decrease in VAS and the ability to stand and ambulate $>15$ minutes were considered to be successful endpoints. All patients had bilateral decompression with L4/5 (34/42) being most common, followed by L3/4 (23/42), L5/S1 (11/42) and L2/L3 (6/42). The majority of patients underwent 2 level decompression (26/42) with 2 patients having 3 level bilateral decompression. A typical pre and post procedure epidurogram is demonstrated in Fig. 1. The remaining patients (7/42) had single level bilateral procedures.

The pre-procedure VAS was reported as $9.6+/-0.42$ with standing and ambulation. This was significantly decreased to $5.8 \pm 2.5$ at 30 days post procedure. All patients reported functional impairment that affected their lives prior to the procedure while $71 \%(n=30)$ perceived an improvement in function following mild. One patient (1/42) reported the ability to walk for $>15$ minutes without symptoms of neurogenic claudication prior to mild versus $60 \%(25 / 42)$ and $73 \%$ (31/42) who reported being able to ambulate and stand upright respectively for > 15 minutes following mild (Table 1). No major complications were noted (Table 2) while minor post procedural complaints (Table 3 ) were of short duration. Five patients requested post procedure opioid analgesia. Thirty-six patients reported they would rec-
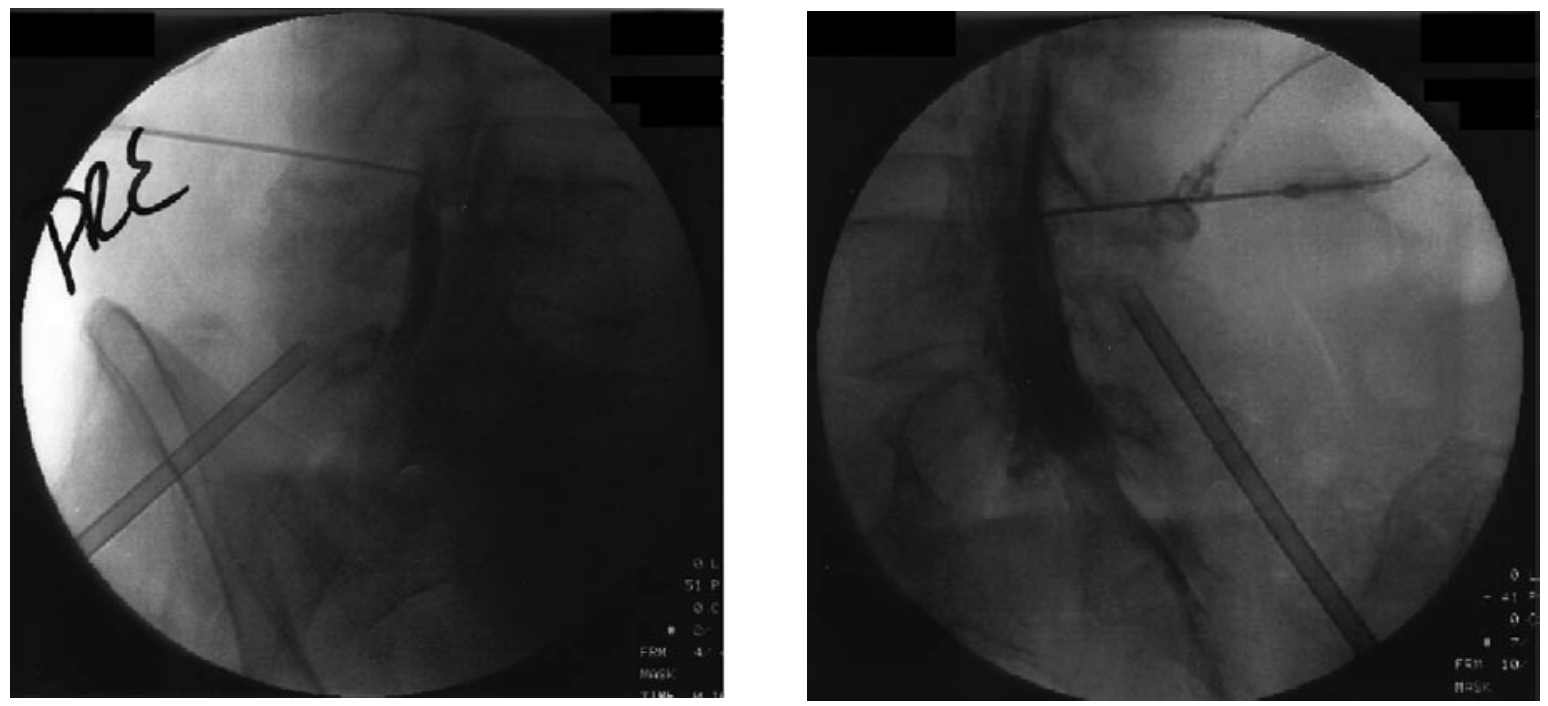

Fig 1. Epidurogram with a $40^{\circ}$ oblique view image a) epidurogram prior to decompression, note lack of contrast spread around the Tuohy needle suggesting L3/4 ligamentum flavum hypertrophy; b) epidurogram (L3/4) following decompression demonstrating markedly improved cephalad contrast spread and increased capacity in the epidural space

Table 1. Chart showing pre and post improvements.

\begin{tabular}{|c|c|c|c|c|c|c|c||}
\hline \multicolumn{2}{|c|}{ VAS } & \multicolumn{2}{c|}{ LSS Impact ADL } & \multicolumn{2}{c|}{ Walk >15 min } & \multicolumn{2}{c|}{ Stand > 15 min } \\
\hline Pre & Post & Pre & Post & Pre & Post & Pre & Post \\
\hline $9.6 \pm 0.42$ & $5.8^{*} \pm 2.5$ & $42 / 42$ & $\begin{array}{c}\text { Improved } \\
31 / 42(73 \%)\end{array}$ & $1 / 42(3 \%)$ & $25 / 42(60 \%)$ & $6 / 42(14 \%)$ & $31 / 42(73 \%)$ \\
\hline
\end{tabular}

${ }^{*} P<0.05$ 
Table 2. Chart of major complications.

\begin{tabular}{|c|c|c|}
\hline Major Adverse Events & Pts Requiring Post Procedure Opioids & Pts Who Would Recommend mil \\
\hline None & $5 / 42$ & $36 / 42(86 \%)$ \\
\hline
\end{tabular}

Table 3. Chart of minor adverse events.

\begin{tabular}{|l|c|c|c|c|}
\hline \multicolumn{2}{|c|}{ Minor Adverse Events } & Duration & \multicolumn{2}{c|}{ Intervention Required/Outcome } \\
\hline Soreness at site & $20 / 42$ & 3.8 days & Post procedure opioid & $5 / 24$ \\
\hline Left gluteal pain & $4 / 42$ & 7 days & Piriformis injection / Resolved & $4 / 4$ \\
\hline Bleeding at incision site & $1 / 42$ & 20 hours & \multicolumn{2}{c|}{ None } \\
\hline Back spasm & $1 / 42$ & 7 days & None \\
\hline
\end{tabular}

ommend this procedure, one was unsure and 5 stated they would not recommend the procedure. Eight patients reported marginal improvement (change $<3$ on VAS) yet 4/8 patients in this category still stated they would recommend mild to other patients.

\section{Discussion}

The current study provides descriptive data in 42 patients outlining the common minor adverse events in patients undergoing mild. We believe that this information is useful to clinicians as they discuss the likely postprocedure course with patients who are candidates for mild. In the initial safety study, and the MiDAS 1 study, major adverse events were described as dural tear, spinal fluid leak, epidural hematoma or abscess $(6,12)$. In keeping with the 90 patient safety study by Deer and Kapural (6) and the MiDAS 1 trial (12), there were no major adverse events experienced in our study. The most common complaint in the current report was mild to moderate soreness at the procedure site lasting approximately 3.8 days and requiring opioid supplementation in only $12 \%$ of patients. Additionally, the outpatient nature of the procedure is highlighted by the fact that no patients required overnight observation following mild. Interestingly, the most common follow up procedure required in our review was a piriformis injection for left gluteal pain. It is unclear whether this is coincidental or a result of increased function and improved posture in patients somewhat deconditioned by limited activity secondary to LSS. All patients undergoing piriformis injection had complete resolution of their symptoms and a further sustained reduction in VAS.

In keeping with the MiDAS 1 trial, we found that the L4/L5 level was most commonly indicated for decompression followed by L3/L4 (12). Also like MiDAS 1, all levels identified required bilateral decompression with the largest percentage of patients requiring the procedure for 2 spinal levels (12). The improvement in contrast flow, the current procedural endpoint, suggesting successful decompression is demonstrated in Fig. 1.

Chopko and Caraway (12) report initial VAS of 7.3 with a $53 \%$ reduction to 3.7 at 42 days follow up. Our initial patient self-reported VAS was 9.6 with a similar 3.8 reduction in VAS to 5.8 . While the absolute starting VAS numbers are different, these data represent a decrease in reported pain scores of a similar magnitude as the MiDAS 1 study. Approximately $73 \%$ of patients reported improvement with the procedure in our nonstandardized questionnaire of functional status, i.e. the ability to ambulate and stand for greater than 15 minutes. Perhaps the most impressive finding is the fact that $86 \%$ percent of patients reported that they would recommend mild; suggesting that even the few patients who did not feel that they had a significant functional improvement felt the procedure was non-invasive enough to have been worth an unsuccessful trial.

At present there are no clear-cut standards as to what constitutes radiologic spinal stenosis, much less ligamentum flavum hypertrophy $(2,15)$. A recent report identified 191 patients with lumbar spinal stenosis and back pain in a community setting. These authors used spinal canal width of less than $12 \mathrm{~mm}$ as relative stenosis and $<10 \mathrm{~mm}$ as absolute stenosis. In this study ligamentum flavum hypertrophy was identified as a major component of spinal stenosis. Further, it was concluded that acquired spinal stenosis might be present in $20 \%$ of patients less than 40 years old and $47 \%$ of patients in their 60s (2). In keeping with these findings, it has recently been shown that ligamentum flavum thickness varies depending upon spinal level and even which side is examined (15). 
With regard to ligamentum flavum thickness, a recent investigation reported that 2.5-3.0 mm thickness is normal at L3/4 while L4/5 and L5/S1 thickness was 4.0 and $3.5 \mathrm{~mm}$ respectively (15). In the lumbar spinal stenosis group however, L3/4 thickness was approximately $4.0 \mathrm{~mm}$ while $L 4 / 5$ showed the greatest amount of acquired thickening to $4.5 \mathrm{~mm}$ or greater (15). Interestingly the right ligmentum flavum at each level was noted to be thicker than the left in control and stenotic patients (15). The stenotic patients chosen for this study were identified based solely on clinical presentation of low back pain and radicular referred pain in the presence of intermittent claudication (15). In the current study, patients were identified based upon symptoms of spinal stenosis/neurogenic claudication in combination with documented, though not measured, radiologic evidence of spinal stenosis. This stenosis was confirmed by reports from a radiologist or spine surgeon. The lack of documented ligamentum flavum thickening for each patient is a drawback to the current study. Future studies could attempt to standardize the selection criteria of patients for this procedure with vigorous determination of ligamentum flavum thickness perhaps better predicting who will benefit from the procedure.

While the literature concerning radiologic definitions for the diagnosis of spinal stenosis is still evolving, there is an established body of literature with regard to interventional treatment modalities that have been shown to be effective in the treatment of spinal stenosis. For example, caudal epidural steroid injections have been shown to significantly reduce reported pain scores and improve Oswestry Disablity Index scores in $60 \%$ of patients trialed $(4,5)$. Likewise other studies have demonstrated significant reductions in pain scores, improved function and decreased opioid intake with both caudal and interlaminar lumbar epidural steroid injections (1621). Our study likewise suggests similar reductions in pain scores seen with epidural steroid injections. It remains to be seen if the duration of the treatment effect of mild is similar to or longer than traditional injective therapy as no follow-up study has yet been published following patients more than 42 days. Current studies are ongoing to address this concern.

Lysis of adhesions has also been suggested as a possible treatment for pain of lumbar origin. A recent study compared caudal epidural steroid injections with percutaneous adhesiolysis. In this study, lysis of adhesions resulted in significant decreases in numeric pain rating scores (NPR) and improved function in $76 \%$ of patients at one year (22). In this report the results were compared with efficacy of caudal epidural steroid injections at one year. While caudal epidural steroid injections have been shown to decrease NPR scores and improve functional capacity for approximately 30 weeks, the results at one year were inferior to those patients who underwent adhesiolysis $(4,22)$. The positive results for adhesiolysis were not limited to spinal stenosis patients as patients with low back pain and radiculitis as well as patients who had undergone spinal surgery all were shown to have significant improvement following adhesiolysis (23-25).

Adhesiolysis can also be accomplished through the endoscopic route. A recent meta-analysis suggested that there is intermediate evidence to support the use of spinal endoscopic lysis of adhesions in patients who have failed percutaneous adhesiolysis (26). While in-depth discussion of this technique is beyond the current focus, excellent reviews are available $(25,26)$. Future studies comparing the mild procedure with treatments such as percutaneous lysis of adhesions for efficacy, duration of treatment effect, and improvement in functional measures will clearly be necessary.

Opioid therapy remains a mainstay in the non-interventional treatment of lumbar spinal pain in general and as such, pain secondary to spinal stenosis. While interventional techniques previously mentioned have clearly been shown to decrease NPR scores and increase function, often patients require additional analgesia (18). Despite widespread use for low back pain, there is still controversy as to the effectiveness of oral opioids of lumbar spinal pain (11). Clearly close monitoring and appropriate drug selection, proper dosing and administration, as well as clear treatment goals are necessary when initiating this treatment $(9,10,11,27)$.

\section{Conclusion}

The results of the current study suggest that minor adverse events with mild consist mainly of soreness at the procedure site which is self-limiting, infrequently requiring additional procedures or even post-procedure opioid as an intervention. In keeping with other reports, the procedure appears to offer a safe and effective alternative to patients suffering from LSS. Clearly prospective, randomized trials comparing safety and efficacy of mild to other established treatments for spinal stenosis will be necessary. 


\section{Disclosures}

This case review study was IRB approved. The authors received no financial support for the study. Richard Lingreen MD, serves as a faculty trainer for the technique.

\section{Acknowledgments}

The authors wish to thank the editorial board of Pain Physician for review and criticism in improving the manuscript.

\section{References}

1. Information on the diagnosis and treatment of spinal stenosis. www.spinalstenosis.org

2. Kalichman L, Cole R, Kim DH, Ling L. Spinal stenosis prevalence and association with symptoms: The Framingham study. Spine J 2009; 9:545-550.

3. Weinstein JN,Tosterson TD, Lurie JD, Tosteson AN, Blood E, Hanscom B, Herkowitz H, Cammisa F, Albert T, Boden SD, Hilibrand A, Goldberg H, Berven S, An H; SPORT Investigators. Surgical versus nonsurgical therapy for lumbar spinal stenosis. $N$ Eng J Med 2008; 358:794-810.

4. Manchikanti L, Cash KA, McManus CD, Pampati V, Abdi S. Preliminary results of randomized, equivalence trial of fluoroscopic caudal epidural injections in managing chronic low back pain: Part 4. Spinal stenosis. Pain Physician 2008; 11:833-848.

5. Manchikanti L, Singh V, Falco FJE, Cash KA, Pampati V. Evaluation of the effectiveness of lumbar interlaminar epidural injections in managing chronic pain of lumbar disc herniation or radiculitis: $\mathrm{A}$ randomized, double-blind, controlled trial. Pain Physician 2010; 13:343-355.

6. Deer TR, Kapural L. New image-guided ultra-minimally invasive lumbar decompression method: The mild $\AA$ procedure. Pain Physician 2010; 13:35-41.

7. Frey ME, Manchikanti L, Benyamin RM, Schultz DM, Smith HS, Cohen SP. Spinal cord stimulation for patients with failed back surgery syndrome: A systematic review. Pain Physician 2009; 12:379-397.

8. Patel VB, Manchikanti L, Singh V, Schultz DM, Hayek SM, Smith HS. Systematic review of intrathecal infusion systems for long-term management of chronic non-cancer pain. Pain Physician 2009; 12:345-360.

9. Trescot AM, Helm S, Hansen H, Benyamin R, Adlaka R, Patel S, Manchikanti L. Opioids in the management of chronic non-cancer pain: An update of American Society of Interventional Pain Physicians' (ASIPP) guidelines. Pain Physician 2008; 11:S5-S62.

10. Trescot AM, Datta S, Lee M, Hansen H. Opioid pharmacology. Pain Physician
2008; 11:S133-S154.

11. Trescot AM, Datta S, Glaser S, Sehgal N, Hansen H, Benyamin R, Patel S. Effectiveness of opioids in the treatment of chronic non-cancer pain. Pain Physician 2008; 11:S181-S200.

12. Chopko B, Caraway DL. MiDAS 1 (mild Decompression Alternative to Open Surgery): A preliminary report of a prospective, multi-centered clinical study. Pain Physician 2010;13:369-378.

13. Todd KH, Funk KG, Funk JP, Bonacci R. Clinical significance of reported change in pain severity. Ann Emerg Med 1996; 27:485-489.

14. Zolman JF. Biostatistics: Experimental design and statistical inference. Oxford University Press, New York, 1993, pp 153155 .

15. Abbas J, Hamound K, Youssef MM, May H, Hay O, Medlej B, Peled N, Hershkovitz I. Ligamentum flavum thickness in normal and stenotic lumbar spines. Spine 2010; 35:1225-1230.

16. Manchikanti L, Cash KA, McManus CD, Pampati V, Smith HS. Preliminary results of randomized, equivalence trial of fluoroscopic caudal epidural injections in managing chronic low back pain: Part 1. Discogenic pain without disc herniation or radiculitis. Pain Physician 2008; 11:785800.

17. Manchikanti L, Singh V, Cash KA, Pampati V, Damron KS, Boswell MV. Preliminary results of randomized, equivalence trial of fluoroscopic caudal epidural injections in managing chronic low back pain: Part 2. Disc herniation and radiculitis. Pain Physician 2008; 11:801-815.

18. Manchikanti L, Singh V, Cash KA, Pampati V, Datta S. Preliminary results of randomized, equivalence trial of fluoroscopic caudal epidural injections in managing chronic low back pain: Part 3. Post surgery syndrome. Pain Physician 2008; 11:817-831.

19. Manchikanti L, Cash KA, McManus CD, Pampati V, Benyamin R. Preliminary results of a randomized, double-blind, controlled trial of fluoroscopic lumbar interlaminar epidural injections in managing chronic lumbar discogenic pain without disc herniation or radiculitis. Pain Physician 2010; 13:E279-E292.

20. Conn A, Buenaventura R, Datta S, Abdi $S$, Diwan S. Systematic review of caudal epidural injections in the management of chronic low back pain. Pain Physician 2009; 12:109-135.

21. Parr AT, Diwan S, Abdi S. Lumbar interlaminar epidural injections in managing chronic low back and lower extremity pain: A systematic review. Pain Physician 2009; 12:163-188.

22. Manchikanti L, Cash KA, McManus CD, Pampati V, Singh V, Benyamin RM. The preliminary results of a comparative effectiveness evaluation of adhesiolysis and caudal epidural injections in managing chronic low back pain secondary to spinal stenosis: A randomized, equivalence controlled trial. Pain Physician 2009; 12:E341-E354.

23. Manchikanti L, Singh V, Cash KA, Pampati V, Datta S. A comparative effectiveness evaluation of percutaneous adhesiolysis and epidural steroid injections in managing lumbar post surgery syndrome: $\mathrm{A}$ randomized, equivalence controlled trial. Pain Physician 2009; 12:E355-E368.

24. Manchikanti L, Pampati V, Cash KA. Protocol for evaluation of the comparative effectiveness of percutaneous adhesiolysis and caudal epidural steroid injections in low back and/or lower extremity pain without post surgery syndrome or spinal stenosis. Pain Physician 2010; 13:E91E110.

25. Epter RS, Helm S, Hayek SM, Benyamin RM, Smith HS, Abdi S. Systematic review of percutaneous adhesiolysis and management of chronic low back pain in post lumbar surgery syndrome. Pain Physician 2009; 12:361-378.

26. Hayek SM, Helm S, Benyamin RM, Singh $\mathrm{V}$, Bryce DA, Smith HS. Effectiveness of spinal endoscopic adhesiolysis in post lumbar surgery syndrome: A systematic review. Pain Physician 2009; 12:419-435

27. Manchikanti L, Atluri S, Trescot AM, Giordano J. Monitoring opioid adherence in chronic pain patients: Tools, techniques and utility. Pain Physician 2008; 11:S155S180. 